\title{
Neural Network Reference Model Control for Inverted Pendulum Based on Simulink
}

\author{
Haiyan Wang \\ Changchun University of science and technology, Changchun 130000, China \\ 13742883@qq.com
}

Keywords: control system,inverted pendulum, reference model control,simulation

\begin{abstract}
This paper sets the mathematical model of the single inverted pendulum,and analyzed the imperfection of PID control algorithm for inverted pendulum. Based on this case, the neural model reference control method is discussed in the paper. With the design of the inverted pendulum system with a model-reference neural controller,system' $\mathrm{s}$ performance and result are analyzed based on the model-reference neural controller in Matlab / Simulink environment.
\end{abstract}

\section{1. the introduction}

Inverted pendulum system is a typical high order time, unstable system.At present, the inverted pendulum as the controlled object control method study emerged a lot of achievements, such as based on PID control and LQR control, neural network control, state feedback control and fuzzy control inverted pendulum control research.In the inverted pendulum control system based on PID, because $\mathrm{K}, \mathrm{p}$ and $\mathrm{K} \mathrm{d}$ parameters is larger, resulting in the actual project amplifier has a high requirement, at the same time when excessive destabilize the system.In view of the shortcomings of PID control and the limitation of actual engineering, this article USES the Reference neural network combined with the Model control method to control the inverted pendulum system, and use the Matlab toolbox "the Model Reference Controller" module to carry out the simulation experiments.

\section{The single-stage inverted pendulum mathematical model is established in this paper}

Single-stage inverted pendulum system and ignore the various friction and air resistance, can abstract for cars and homogeneous system composed of thin rod, as shown in figure 1.

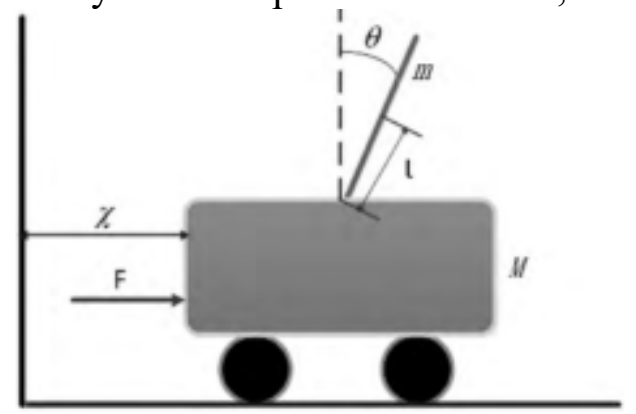

Figure 1 single-stage inverted pendulum system structure

In figure $1, \mathrm{~F}$ for the role of external forces on the car, $\mathrm{M}$ is the quality of the car, to the quality of the swinging rod, 1 is the length of the pendulum rod axis of rotation to the center of mass of, $\mathrm{x}$ is the position of the car, theta is the Angle of swinging rod in vertical direction.In this paper in the system to select the car position vector $\mathrm{x}$ and swinging rod Angle theta as the generalized coordinates, and by using Lagrange the second equation, the equilibrium position linearization available system dynamic equation as shown in type (1) :

$$
\theta^{\prime \prime}=\frac{3 g}{41} \theta+\frac{3}{41} x^{\prime \prime}
$$


With acceleration as the input, the Angle of swinging rod as output, plug as shown in table 1 of the actual data of the inverted pendulum system components, the system transfer function such as type (2) shown below:

$$
G(s)=\frac{\theta(s)}{u(s)}=\frac{3}{s^{2}-29.4}
$$

The type (2) shows that the car system is an unstable system.

Actual parameters in table 1

\begin{tabular}{|c|c|c|}
\hline $\mathrm{M}$ & The car quality & $0.5 \mathrm{~kg}$ \\
\hline $\mathrm{m}$ & Swinging rod quality & $0.25 \mathrm{~kg}$ \\
\hline 1 & $\begin{array}{c}\text { The length of the pendulum rod axis of } \\
\text { rotation to the rod center of mass }\end{array}$ & $0.25 \mathrm{~m}$ \\
\hline $\mathrm{b}$ & The car the coefficient of friction & 0.1 \\
\hline $\mathrm{g}$ & Acceleration of gravity & $9.8 \mathrm{~kg} . \mathrm{m} / \mathrm{s} 2$ \\
\hline
\end{tabular}

\section{3. reference model control principle and single-stage inverted pendulum control}

\section{1 the neural network model reference adaptive control theory}

Reference model control system by the reference model and adjustable system and adaptive mechanism of three parts, the adjustable part includes the controlled object, neural network controller and neural network identifier, as shown in figure 2.

In this system, using a reference model for auxiliary system. System of the input signal at the same time on the controller and the reference model, the output of the reference model is the expected output of the system.

If the reference model and adjustable output, or the process of the system directly is presupposed, and get the error signal e ( $\mathrm{t}) 1$ and $2 \mathrm{e}(\mathrm{t})$, then the system according to the principle of adaptive feedback effect, according to the direction of reduce error correction or updating control, in order to make the system of the actual performance of the performance indicators at or close to hope.

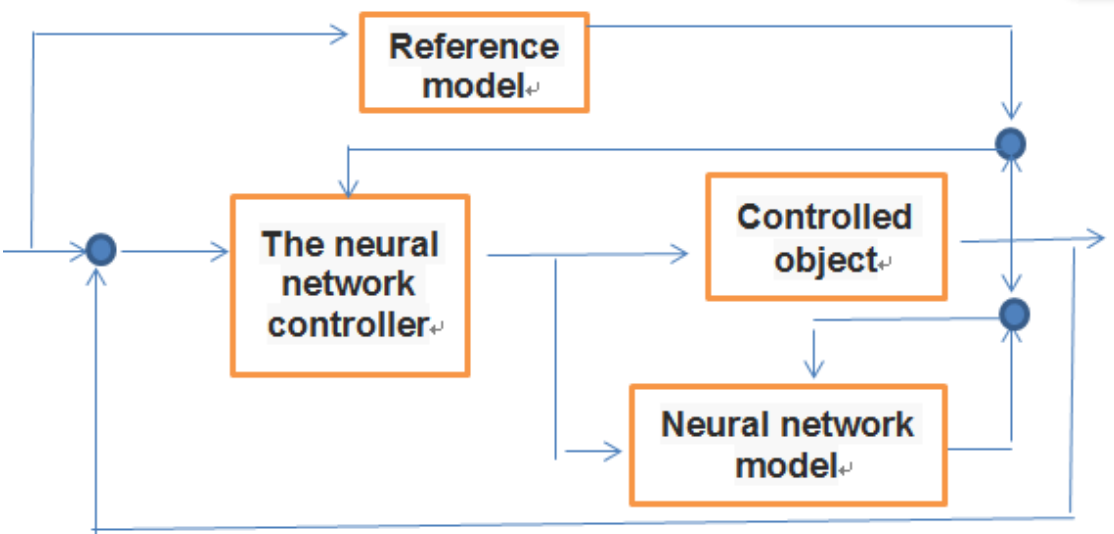

Figure 2 neural network reference model of the controller structure

\section{2 to establish a single inverted pendulum with Simulink simulation system}

Reference model designed neural network controller and neural network model, the library reference model using Simulink in Matlab neural network controller.Build Simulink control of inverted pendulum model first, and then establish a system control model of reference is shown in figure 3.

\section{3 reference control model of Simulink simulation process}

Detailed simulation process can be divided into the following three stages

1) neural network identification system. Double click on the Model Reference Controller module to produce a new window, in the first place for system identification. Setting simulation parameters, choose the appropriate simulation algorithm, click the generate Train Data button, to generate the training Data. Then click on the "Train" the Network to Train the neural Network. In the process of 
system identification neural network can adjust the number of hidden layer and repetitive training, until satisfactory results.

2) model reference control.In model reference control window, click the Generate Training Data button and Train controller button, start Training the neural network controller.Network program will be a piece of data input, and to specify the number of iterations network. This process, the controller USES the dynamic back propagation algorithm, will spend a lot of time at a price.

3) the system simulation.Return to the Simulink model, setting simulation parameters, including the simulation of $50 \mathrm{~s}$, and then choose Start command to Start the simulation.

Figure 3 for the above process flow chart. In model reference control stage also need like system identification stage to judge if not satisfied with the results, can adopt the same way to adjust.

From the result of simulation shows that single stage inverted pendulum reference model of control system of random step signal has strong tracking effect, if the results of simulation is not accurate, recommends for neural network training, mainly by adjusting the number of hidden layer and the number of training.

But as the number of hidden layer and with the increase of number of training system cost will increase, so the general need to compromise considering these parameters.

\section{4、Conclusion}

Control by using neural network toolbox reference model, a simulation experiment, makes the performance of the original system had the very big improvement, system stability, accuracy and rapidity had the very big difference.

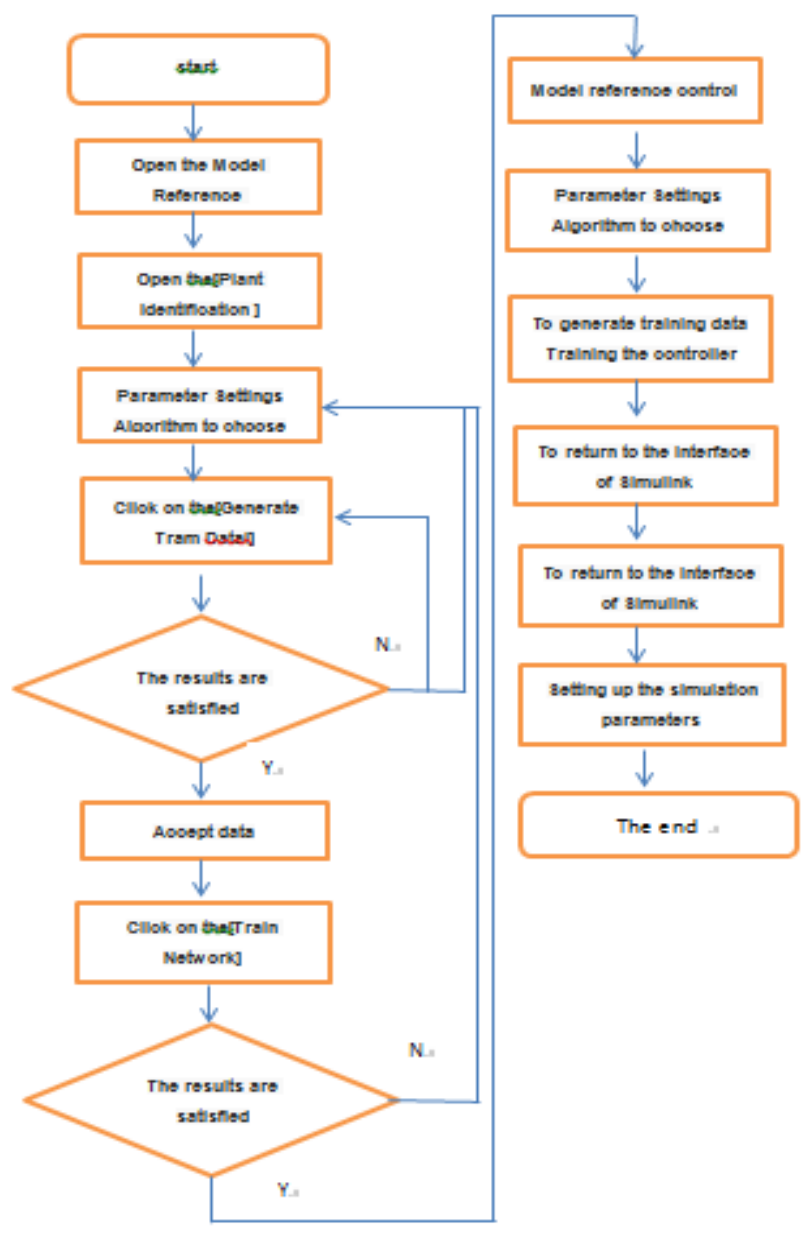

Figure 3 simulation process flow chart 


\section{References}

[1] xiao-bing hu, cheng-zhong liu, xiao-jun zhao. Circular level inverted pendulum system, single neuron PID control simulation [J]. Journal of gansu agricultural university, 2016, (5) : 132-136.

[2] Jiang Hongfa. Design and the optimal control of inverted pendulum based on MATLAB simulation [J]. Journal of intelligent robot, 2016, (10) : 47-50.

[3] the king to tzu chi, Yang Yunong. Level of rotational inverted pendulum and their research and realization of the control device [J]. Journal of southwest normal university (natural science edition), 2016, (8) : 145-150.

Practice.

[4] yellow single-stage rotary inverted pendulum [J]. The design and implementation of science and technology plaza, 2016, (6) : 41-44.

[5] PuJianBo Peng Xiaole, Yin Yandong, Dai Mengxi, Fan Wenrui. Comparative study of the inverted pendulum control method [J]. Industrial instrumentation and automation devices, 2016, (02) : 11-15. 